\title{
Feuermanagement mit Geographischen Informationssystemen
}

\begin{abstract}
Wildfire management requires the analysis of a wide spectrum of data, ranging from physical to socio-economic parameters, of which the majority is spatially distributed. The use of Geographical Information Systems (GIS) can help to manage these heterogeneous data, as has been demonstrated with several projects carried out at the Spatial Data Handling Division of the Department of Geography, University of Zurich. A framework for an integrated wildfire risk assessment is outlined, which allows the estimation of the expected fire damage both for individual objects as well as for all potential starting points. This framework also considers fire warning and fire fighting activities which influence the risk situation.
\end{abstract}

\section{Einleitung}

Waldbrände sind komplexe, dynamische Prozesse, welche in vielen Gebieten einen wichtigen Bestandteil der natürlichen Vegetationsdynamik bilden. So können sie zur Mosaikbildung, zur Artenvielfalt und zum Verjüngungsproze $ß$ von Vegetationsgesellschaften beitragen. Andererseits ist der Mensch seit je gezwungen, sich vor der zerstörerischen Wirkung von Waldbränden zu schützen. Heute führt die in vielen Ländern zunehmende Nutzung brandgefährdeter Gebiete sowie die Erhöhung der Brandgefahr durch menschliche Aktivitäten und technische Anlagen zu einer Konfliktsituation, welche einen umfassenden Umgang mit der Waldbrandproblematik erfordert. So sollen die negativen Folgen von Waldbränden so weit wie möglich reduziert, ihre erwünschten Auswirkungen jedoch nach Möglichkeit beibehalten werden. Ein solcher Ansatz wird als Feuermanagement bezeichnet.

Aufgrund der Interdisziplinarität des Feuermanagements und des ausgeprägten Raumbezugs der verwendeten Informationen erscheinen gerade Geographische Informationssysteme (GIS) als geeignetes Werkzeug für die Behandlung der Waldbrandproblematik. Am Geographischen Institut der Universität Zürich ist deshalb ein Projekt im Gange, welches sich mit Anwendungsmöglichkeiten von GIS im Feuermanagement befaßt. Die hauptsächlichen Untersuchungsgebiete stellen der Schweizerische Nationalpark und das Tessin dar. Die Projektziele orientieren sich jedoch auch an der Waldbrandsituation in anderen Ländern innerhalb und außerhalb Europas. Hier sollen einige der im Rahmen dieses Projekts durchgeführten Arbeiten vorgestellt werden.

\section{Grundlagen}

\subsection{Waldbrand}

Unter dem Begriff "Waldbrand» verstehen wir alle unkontrollierten Brände, welche in natürlicher oder naturnaher Vegetation auftreten. Darunter fallen also beispielsweise auch Busch- oder Grasbrände (im Englischen spricht man treffender von wildfires). Wie die letzten Jahre gezeigt haben, ist dabei die Grenze zu Bränden in städtischen Gebieten oft fließend, bedingt durch die gerade in mediterranen Klimagebieten zunehmende Verzahnung von Siedlungsgebieten mit naturnahen Räumen. Waldbrände können die unterschiedlichsten Ursachen (wobei der Mensch und seltener Blitzschlag zu den Hauptauslösern zählen) sowie Erscheinungsformen aufweisen. Abb. 1 zeigt die wichtigsten Typen von Waldbränden mit ihren gegenseitigen Abhängigkeiten.

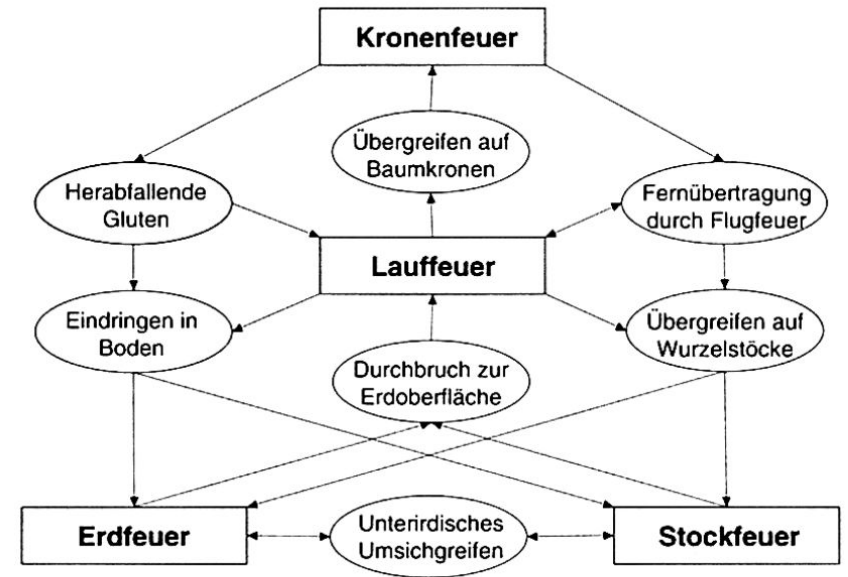

Abb.1 Waldbrandtypen und inre Abhängigkeiten (nach Jecklin und Schöb, 1993).

Andreas Bachmann, dipl. Geogr., Reto Schöning, dipl. Geogr., Britta Allgöwer, Dr. sc. tech., Geographisches Institut der Universität Zürich, Winterthurerstraße 190, 8057 Zürich (bachmann,reto,britta)@geo.unizh.ch 
Die am häufigsten auftretende Form stellen die Laufoder Bodenfeuer dar. Sie bilden fast immer die Anfangsform eines Waldbrandes. Das Feuer breitet sich dabei in der Streu-, Kraut- und Strauchschicht aus. Wenn die Intensität genügend groß ist, können die Flammen eines Bodenfeuers auf den Kronenraum eines Waldes übergreifen und damit ein Kronenfeuer auslösen. Durch starke Luftströmungen, die von Winden, aber auch durch die Konvektion von Bränden erzeugt werden, können glühende oder brennende Teile des Brandgutes durch die Luft getragen werden und neue Feuer entzünden. Solche Flugfeuer können beachtliche Distanzen überwinden (in Eukalyptuswäldern bis zu Dutzenden von Kilometern). Als besonders heimtückisch erweisen sich auch Erdoder Stockfeuer. Meist entstehen diese direkt durch Blitzschlag oder werden durch ein oberirdisches Feuer ausgelöst. Sie breiten sich unterirdisch im organischen Material aus. Da sie häufig nur glimmen und fast keinen Rauch entwickeln, sind sie nur schwer erkennbar. Wie der Waldbrand im Walliser Pfynwald (Frühjahr 1996) gezeigt hat, können solche Erdfeuer noch Wochen später an einem anderen Ort wieder Lauffeuer entfachen.

$\mathrm{Zu}$ den am stärksten von Waldbränden betroffenen Gebieten gehören jene mit mediterranen und borealen Klimata. Aber auch in der Schweiz treten jedes Jahr Brände auf. Die Waldbrandstatistik der Eidgenössischen Forstdirektion verzeichnet zwischen 1967 und 1991 einen Durchschnitt von 151 Brandereignissen mit einer Totalfläche von 335 ha pro Jahr (EIDG. FORSTDIREKTION 1992). Der Kanton Tessin war im genannten Zeitraum mit 45\% der Ereignisse am stärksten betroffen, gefolgt von den Kantonen Graubünden und Bern.

\subsection{Feuermanagement}

In vielen Gebieten der Welt erfordert die hohe Waldbrandaktivität in Verbindung mit wachsenden Nutzungsansprüchen an brandgefährdete Flächen ein umfassendes System für die Begrenzung der negativen ökonomischen und ökologischen Auswirkungen von Waldbränden. Dieses wird als Feuermanagement bezeichnet (engl. fire management). SIMARD (1976) definiert Feuermanagement so:

The application of management, physical, and ecological principles to the management of the wildland fire process so as to render the impact of wildland fire on the natural resource base, the ecosystem, and the environment consistent with the goals of the managing organization. Wildland fire management includes the traditional fire control related activities (suppression, detection, etc.) as well as broader relationships between fire and wildland management, such as prescribed fire and fuel management.

Von entscheidender Bedeutung im Feuermanagement ist die langfristige und vernetzte Betrachtungsweise, welche die beteiligten ökologischen und sozioökonomischen Prozesse über große Zeiträume zu berücksichtigen versuchen sollte. Die Waldbrandproblematik muß im größeren Zusammenhang sowohl der Nutzungsziele für das betrachtete Gebiet wie auch der Rolle von Feuer in dessen natürlicher Vegetationsdynamik betrachtet werden. Feuer wird nicht generell als Gefahr angesehen, die es unter allen Umständen zu verhindern gilt, sondern es kann auch systematisch eingesetzt werden, um gewisse Ziele (z. B. die Reduktion gefährlicher Brandgutanhäufungen) zu erreichen. Die zum Teil umfangreichen Programme "verschriebener Brände» (engl. prescribed fires) vor allem in den USA, Kanada und Australien sind ein bekannter Ausdruck davon. Dabei werden Brände künstlich ausgelöst oder ungeplant ausgebrochene Brände toleriert, sofern bestimmte, klar definierte (meist wetterund brandgutbezogene) Bedingungen erfüllt sind. Dieses Teilgebiet des Feuermanagements macht besonders deutlich, wie wichtig ein ausreichendes Verständnis der beteiligten Prozesse ist. Entsprechend wird gerade in den genannten Ländern sowie vermehrt auch in Europa die Forschung zur Entstehung, zum Verhalten und zu den Folgen von Waldbränden intensiv vorangetrieben. Auch wenn man noch weit von einem vollständigen Verständnis des Phänomens Waldbrand entfernt ist, so erlaubt der heutige Wissensstand doch einen sachlichen Umgang mit Waldbränden und eine Abkehr von der durch Volksweisheiten wie «Wer mit dem Feuer spielt, verbrennt darin» geprägten Sicht.

Die umfassende Betrachtungsweise des Feuermanagements erfordert die Integration von Beiträgen aus den verschiedensten Fachrichtungen. Dabei fallen große Datenmengen an, welche größtenteils einen direkten oder indirekten Raumbezug aufweisen. Es erstaunt deshalb nicht, daß Geographische Informationssysteme im Feuermanagement eine wichtige Rolle spielen. Mit GIS ist ein Integrationswerkzeug vorhanden, mit welchem Daten aus den verschiedenen Fachgebieten miteinander verknüpft und ausgewertet werden können. Von besonderer Bedeutung für die Verwendung im Feuermanagement ist dabei die Möglichkeit der Einbindung von Modellen, beispielsweise zum Brandverhalten. Diese Modelle ergänzen einerseits die GIS-Werkzeuge für die räumliche Analyse um eine entscheidende fachspezifische Komponente, andererseits ergeben sich durch die Kombination mit den allgemeinen Analysewerkzeugen völlig neue Verwendungsmöglichkeiten. In Kapitel 3 sollen Möglichkeiten der Verwendung von GIS im Feuermanagement anhand ausgewählter Beispiele aus dem Schweizerischen Nationalpark und dem Tessin illustriert werden. In Kapitel 4 werden die neuen Perspektiven für die Feuermanagementplanung skizziert, welche sich durch die Kombination von spezifischen Waldbrandmodellen mit den Analysemethoden eines GIS eröffnen.

\subsection{Waldbrandmodellierung}

Der große Bedarf aus der Praxis nach Methoden der Simulation und Prognose von Waldbränden bewirkte die Entwicklung einer Vielzahl von Modellen, welche verschiedenste Aspekte der Waldbrandproblematik betrachten. Von großer Bedeutung war und ist die Simulation des Waldbrandverhaltens, d. h. die Modellierung der 
Feuerausbreitung und der Feuerintensität. Die Ausbreitung eines Feuers, selbst durch ein homogenes Brandgutbett und unter konstanten Bedingungen, ist jedoch ein sehr komplexer Prozeß. Die vollständige Formulierung eines Modells zum Waldbrandverhalten und dessen numerische Lösung, wie sie von Wissenschaftern angestrebt wird, steht den Forderungen der Praxis gegenüber, welche einfach verwendbare, effiziente und zuverlässige Modelle für den operationellen Einsatz benötigen. Nach dem Grad der Berücksichtigung physikalischer Prinzipien können die Modelle unterschieden werden in empirische, semiempirische oder physikalische Ansätze. Das wohl am weitesten verbreitete ist das semiempirische Modell von ROTHERMEL (1972), welches in den 60er und $70 \mathrm{er}$ Jahren in den USA entwickelt wurde. Mit Hilfe dieses Modells kann das lokale Brandverhalten von Lauffeuern berechnet werden. Benötigt werden Daten zum Gelände, zum Brandgut, zum Wind und zur Brandgutfeuchtigkeit (siehe Abb. 2).

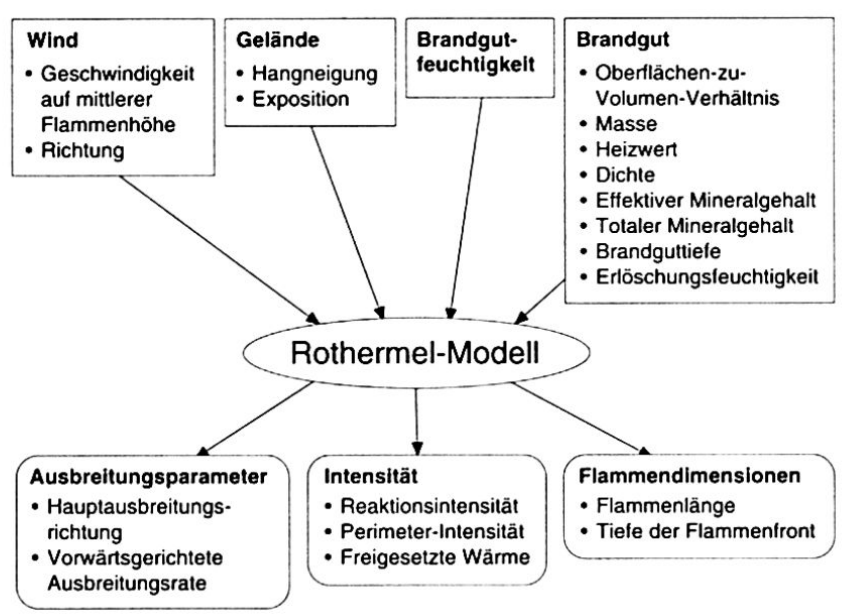

Abb.2 Eingangsdaten und Resultate des RothermelModells.

Als wichtigste Resultate liefert das Modell die Hauptausbreitungsrichtung und Ausbreitungsrate.

Durch die flächendeckende Berechnung des lokalen Brandverhaltens ist es möglich, die Ausbreitung eines Brandes zu simulieren. Dazu benötigt man ein Modell zur geometrischen Beschreibung der Brandform. Am häufigsten verwendet wird das Modell von ANDERSON (1983). Es beschreibt die Form eines Brandes, der sich in einem homogenen, ebenen Brandgutbett unter gleichförmigen Bedingungen ausbreitet, durch zwei Halbellipsen, deren Parameter von der Windgeschwindigkeit abhängen.

\section{Beispiele für die Verwendung von GIS im Feuermanagement}

\section{1 Überblick}

Neben der wichtigen Funktion der zentralen Verwaltung der für das Feuermanagement spezifischen Daten und ihrer flexiblen Visualisierung bietet ein GIS zusätzlich die Möglichkeiten der Analyse und Modellierung. Anwendungen dieser Funktionalitäten sind zum Beispiel Ausbreitungssimulationen, flächendeckende Berechnung des Brandverhaltens, Maßnahmenplanung, Analyse der Brandgeschichte und der Brandgefahr usw. Am Geographischen Institut der Universität Zürich sind mehrere Arbeiten durchgeführt worden, welche die Funktionalität eines GIS für waldbrandspezifische Fragestellungen verwendet haben. Sie sollen hier beispielhaft aufzeigen, welche Möglichkeiten sich mit dem Einsatz von GIS ergeben.

\subsection{Waldbrandmodellierung}

Wie erwähnt, ist die Modellierung des Waldbrandverhaltens von großem Interesse für das Feuermanagement. SCHÖNING (1996a) hat eine Prototyp-Applikation namens Sparks entwickelt, welche zum Ziel hatte, einerseits die Eignung eines kommerziellen GIS (ARC/INFO) als Plattform für die Modellierung zu evaluieren und andererseits die Möglichkeiten von bestehenden GIS-Funktionalitäten bei der Analyse und Visualisierung von waldbrandspezifischen Daten und Prozessen aufzuzeigen. Sparks verfügt über eine graphische Benutzeroberfläche, welche die Datenverwaltung, die interaktive Analyse und Visualisierung, die Modellsteuerung sowie die Bearbeitung der Brandgutdaten ermöglicht. Abb. 3 zeigt die Menüoberfläche von Sparks.

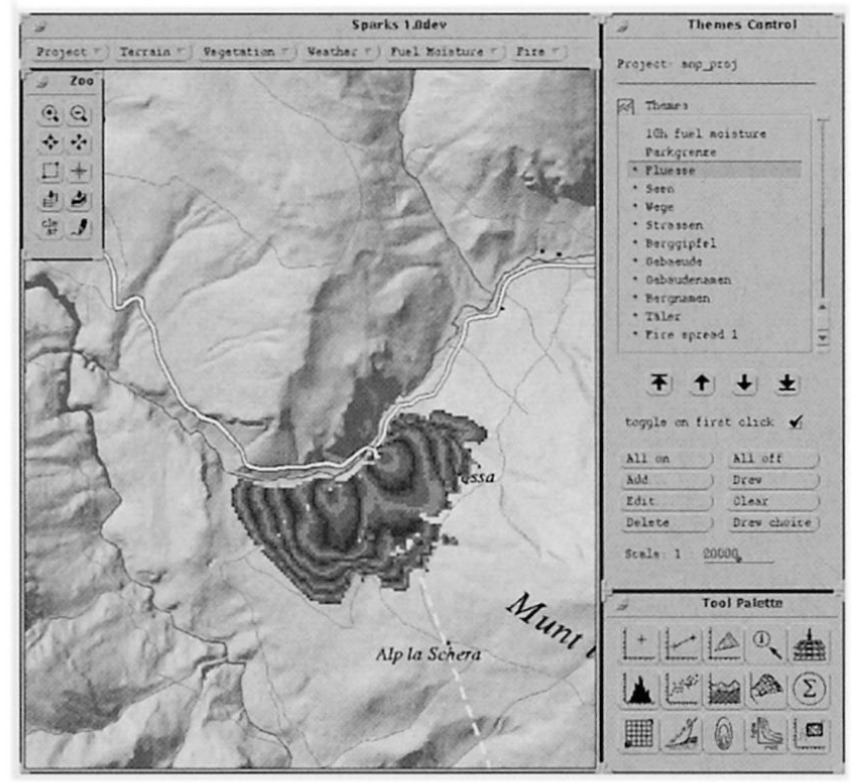

Abb.3 Menüoberfläche von Sparks mit einer Ausbreitungssimulation. 
Kern der Applikation sind die Berechnung des Waldbrandverhaltens nach Rothermel sowie die Ausbreitungssimulation. Die Gleichungen des Rothermel-Modells wurden im ARC/INFO-Modul GRID zur Verarbeitung von Rasterdaten implementiert. Wenn die Eingabedaten flächendeckend in Form von Rasterdatensätzen vorliegen, wird das lokale Brandverhalten für jedes einzelne Raumelement berechnet. Das Resultat stellt die Grundlage für die Ausbreitungssimulation dar. Ausgehend von frei wählbaren Startpunkten und unter Verwendung von Andersons Modell für die Brandform (ANDERson 1983), wird für alle Zellen die Ausbreitungszeit berechnet. Das Resultat ist ein Rasterdatensatz, der für jede Zelle die Zeit angibt, innerhalb welcher sie von der Feuerfront erreicht wird.

\subsection{Stichprobenplanung im Rahmen von Brandgutdatenerhebungen}

Das Modellieren des Waldbrandverhalten sowie die Ausbreitungssimulation erfordern die flächendeckende Kenntnis der Brandguteigenschaften. Unter dem Begriff Brandgut versteht man alles organische, lebendige oder tote Material, in, auf oder über dem Boden, welches sich entzünden und brennen kann. Im Rothermel-Modell wird das Brandgut mit Hilfe von sogenannten Brandgutmodellen, welche durch Brandgutparameter beschrieben werden, einbezogen (siehe Tab.1). Im Normalfall werden nicht alle Parameter erhoben, sondern einige konstant gehalten, da entweder ihr Einfluß unbedeutend oder ihre Variation relativ gering ist.

\begin{tabular}{|c|c|c|}
\hline Parameter ${ }^{\circ}$ & Mess & \\
\hline \multicolumn{3}{|c|}{ Oberflächen-zu-Volumen-Verhältnis $\left(\mathrm{m}^{-1}\right)$} \\
\hline Totes Material: & $\begin{array}{l}\varnothing<0,6 \mathrm{~cm} \\
0,6<\varnothing<2,5 \mathrm{~cm} \\
2,5<\varnothing<7,6 \mathrm{~cm}\end{array}$ & $\begin{array}{l}\otimes \\
\square \\
\square\end{array}$ \\
\hline Lebendes Material: & $\begin{array}{l}\text { krautig } \\
\text { verholzt }\end{array}$ & \\
\hline \multicolumn{3}{|l|}{ Biomasse $\left(\mathrm{kg} \mathrm{m}^{-2}\right)$} \\
\hline Totes Material: & $\begin{array}{l}\varnothing<0,6 \mathrm{~cm} \\
0,6<\varnothing<2,5 \mathrm{~cm} \\
2,5<\varnothing<7,6 \mathrm{~cm}\end{array}$ & $\begin{array}{l}\bigotimes \\
\text { } \\
\text { 冈 }\end{array}$ \\
\hline Lebendes Material: & $\begin{array}{l}\text { krautig } \\
\text { verholzt }\end{array}$ & 凶 \\
\hline \multicolumn{2}{|l|}{ Brandguttiefe (m) } & 凶 \\
\hline \multicolumn{2}{|l|}{ Erdlöschungsfeuchtigkeit (\%) } & $凶$ \\
\hline \multicolumn{2}{|c|}{ Dichte der Brandgutpartikel $\left(\mathrm{kg} \mathrm{m}^{-3}\right)$} & $\square$ \\
\hline \multicolumn{2}{|l|}{ Effektiver Mineralgehalt (\%) } & $\square$ \\
\hline \multicolumn{2}{|l|}{ Totaler Mineralgehalt (\%) } & $\square$ \\
\hline \multicolumn{2}{|l|}{ Heizwert $\left(\mathrm{kJ} \mathrm{kg}^{-1}\right)$} & $\square$ \\
\hline
\end{tabular}

Tabelle 1 Brandgutparameter für das Rothermel-Modell (nach Harvey, 1996).
Die Erhebung der benötigten Parameter im Feld ist eine aufwendige und zeitintensive Arbeit. Es ist deshalb von großer Bedeutung, daß die Stichprobenplanung sorgfältig durchgeführt wird. HARVEY hat in seiner Arbeit (1996) eine Methode vorgestellt, die mit Hilfe der systematischen Stratifizierung die Effizienz der Stichprobenerhebung erhöht. Die Methode beruht darauf, das Untersuchungsgebiet aufgrund von Expertenwissen in möglichst homogene Straten aufzuteilen. Mit Hilfe des GIS kann für jedes Stratum ein regelmäßiges Stichprobenraster erzeugt werden, dessen Maschenweite so variiert wird, daß für alle Straten die gleiche Anzahl von Erhebungen anfällt. Nach der Erhebung werden die Straten mittels einer Diskriminanzanalyse getestet und die Mittelwerte pro Brandgutparameter berechnet. Die Methode wurde im Malcantone (TI) mit guten Resultaten getestet. Im Schweizerischen Nationalpark wurde im Sommer 1996 ebenfalls eine Erhebung durchgeführt, die nun als Grundlage für die Waldbrandmodellierung dient.

\subsection{Entscheidungsunterstützung des Feuermanagements}

Die Menge der zu berücksichtigenden Informationen und die Vielzahl der beteiligten Fachgebiete stellen eine der großen Herausforderungen im Feuermanagement dar. Der Einsatz von interaktiven Entscheidungsunterstützungssystemen kann deshalb einen wichtigen Beitrag zur adäquaten Behandlung der Waldbrandproblematik leisten. Diese Systeme verbinden den Einsatz von Modellen mit traditionellen Methoden des Datenzugriffs und der Datenselektion und helfen dem Benutzer bei der Lösung von schlecht strukturierten Problemen. Entscheidungsunterstützende Systeme verfügen über drei zentrale Komponenten: eine Datenbank, eine Modellsammlung und eine Benutzerschnittstelle. Sämtliche Informationen zu den relevanten Teilaspekten des Problemkomplexes werden von der Datenbank verwaltet. In der Modellsammlung werden Funktionen für den Modellaufbau und die Modellanalyse (Modellberechnungen, Szenariotechniken, Sensitivitätsanalyse usw.) zur Verfügung gestellt. Schließlich unterstützt eine interaktive Benutzerschnittstelle den Benutzer bei der Ermittlung einer optimalen Lösung.

Aufgrund ihrer Funktionalität bietet sich die Integration von GIS in ein Entscheidungsunterstützungssystem an. RÜEGSEGGER (1996) hat in ihrer Arbeit aufgezeigt, welche Rolle ein GIS im Feuermanagement spielen könnte. Als Beispiel einer möglichen Managementanwendung wurde die Ausscheidung von Prioritätszonen für die Waldbrandbekämpfung im Untersuchungsgebiet Malcantone gewählt. Die Einteilung der Prioritätszonen richtet sich nach dem Schadenpotential, d. h. dem potentiellen Schaden, welcher durch einen Brand entstehen könnte. Aufgrund der Brandgeschichte und des Waldbrandverhaltens wurde das gesamte Untersuchungsgebiet in fünf Prioritätszonen («sehr geringes» bis «extremes» Schadenpotential) eingeteilt. Die Erfahrung zeigt, $\mathrm{da} ß$ die Vegetation ehemaliger Brandflächen im Tessin 
"feuergefährlicher» ist als diejenige unbeeinflußter Flächen. Die erhöhte Feuergefährlichkeit führt dazu, daß ehemalige Brandflächen häufiger von Bränden betroffen sind, was wiederum die Degradation der Vegetation verstärkt. Die Anlayse der Waldbrandgeschichte beruht auf der räumlichen Analyse ehemaliger Brandflächen sowie auf der statistischen Auswertung der dazugehörigen Attribute. Im Weiteren wurde das Untersuchungsgebiet aufgrund des Waldbrandverhaltens klassiert. Rüegsegger konnte zeigen, daß mit Hilfe eines GIS eine räumlich differenzierte Einteilung von Prioritätszonen möglich ist.

\section{Analyse des Waldbrandrisikos mit GIS}

\section{1 Überblick}

Waldbrände spielen in vielen Gebieten eine wichtige Rolle in der natürlichen Vegetationsdynamik. Bedingt durch die menschliche Nutzung stellen sie aber oft auch ein beträchtliches Gefahrenpotential dar. Bei der Maßnahmenplanung im Feuermanagement müssen beide Aspekte berücksichtigt und einander abwägend gegenübergestellt werden. Die Begrenzung der Gefährdung menschlicher Güter auf ein akzeptables Maß ist dabei in jedem Fall Bedingung. Ein erfolgreiches Feuermanagement erfordert daher eine Methode für die Beurteilung dieser Gefährdung. Die räumliche Analyse des Waldbrandrisikos mit GIS kann dazu einen Beitrag liefern. Der Begriff Risiko bezeichnet ein Maß für eine Gefährdung und umfaßt sowohl das Ausmaß möglicher gefährlicher Ereignisse als auch deren Eintretenswahrscheinlichkeit. Im Fall des Waldbrandrisikos manifestiert sich das Ausmaß in den durch ein Brandereignis verursachten Schäden an gefährdeten Objekten. Als Objekte werden dabei alle für die Risikobeurteilung relevanten, natürlichen und künstlichen Entitäten verstanden, welche durch Waldbrände Schaden erleiden können. Diese unscharfe Beschreibung deutet schon auf die Schwierigkeit hin, den Schadenbegriff in diesem Zusammenhang adäquat zu definieren, geschweige denn der quantitativen Analyse zugänglich zu machen.

Eine Methode für die räumliche Analyse des Waldbrandrisikos sollte unter anderem helfen, die folgenden Fragen zu beantworten:

- Wo befinden sich Zonen, von denen eine große Gefahr ausgeht?

- Welche Objekte sind besonders gefährdet?

- Welcher Gesamtschaden ist in den nächsten zehn Jahren in einem Gebiet zu erwarten?

- Wie werden geplante Managementmaßnahmen (z. B. das Anlegen von Schutzstreifen) die Gefahrensituation beeinflussen?

Bei der Analyse des Waldbrandrisikos muß beachtet werden, daß die Quelle der Gefährdung (mögliche Start- punkte von Waldbränden) von deren Empfängern (den Objekten) räumlich getrennt ist. Die Gefährdung eines Objekts ist abhängig von seiner Nähe zu den potentiellen Startpunkten und von der Wahrscheinlichkeit, daß an diesen Punkten tatsächlich Waldbrände entstehen.

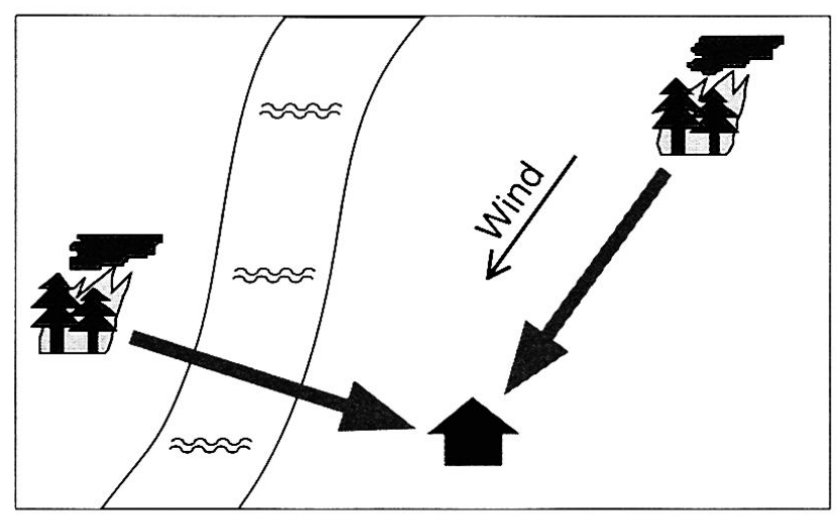

Abb. 4 Unterschiedlicher Einfluß der Distanz auf das Waldbrandrisiko.

Je weiter ein Punkt vom Objekt entfernt ist, desto geringer ist die Wahrscheinlichkeit, daß das Objekt von einem dort entstandenen Waldbrand beeinträchtigt wird. Abb. 4 zeigt, daß diese Wahrscheinlichkeit nur teilweise von der Raumdistanz bestimmt wird, vielmehr jedoch das Ausbreitungsverhalten eines Brandes berücksichtigt werden muß. Dieses wiederum ist von Faktoren wie dem Wetter, der Brandgutverteilung, der Geländeform und dem Vorhandensein von Barrieren abhängig.

Ein weiteres Problem ergibt sich aus der Tatsache, daß jede mit brennbarer Vegetation bedeckte Raumeinheit eine potentielle Quelle von Waldbränden darstellt. Die Gefahrenquelle ist also nicht genau lokalisierbar, sondern mit unterschiedlichen Wahrscheinlichkeiten kontinuierlich im Raum verteilt. Dies stellt einen wichtigen Unterschied zu anderen natürlichen und vor allem technischen Gefahren dar, bei denen die Gefahr von definierten Örtlichkeiten ausgeht (z. B. Fabriken, Verkehrswege) oder bekannte Wirkungsbereiche aufweist (z. B. Hochwasserzonen). Während diese Gefahren meist mit einer beschränkten Anzahl möglicher Szenarien beschrieben werden können, ist dies bei Waldbränden durch die unendliche Menge möglicher Startpunkte nicht möglich.

Im Folgenden wird ein Methodengerüst vorgestellt, welches die genannten Probleme zu lösen versucht und eine umfassende Analyse des Waldbrandrisikos mit Hilfe eines Geographischen Informationssystems ermöglichen soll.

\subsection{Aufbau der Risikoanalyse}

Die Grundstruktur für die Berechnung des Waldbrandrisikos beruht auf der Methode für die Berechnung von technischen Todesfallrisiken von MERZ et al. (1995), 
wobei waldbrandspezifische Ergänzungen gemacht wurden. Die Grundelemente bilden Situationen, Szenarien und gefährdete Objekte.

- Situationen definieren relevante Zustände der Einflußgrößen der Risikoanalyse, z. B. der Meteoparameter oder der menschlichen Aktivitäten.

- Szenarien beschreiben mögliche Brandereignisse. Ein Szenario entspricht einem angenommenen Brandausbruch an einer bestimmten Lokalität in einer definierten Situation.

- Objekte stellen die durch Waldbrände gefährdeten, künstlichen und natürlichen Entitäten dar.

Die Grundlagen für die Risikoanalyse bilden die lokale Ausbruchswahrscheinlichkeit, das Ausbreitungsverhalten des entstehenden Brandes sowie eine Abschätzung der Schädigung von Objekten durch Einwirkung der Flammen. In einer Risikomatrix (Tab. 2) werden die Beziehungen zwischen den Szenarien und den Objekten für eine Situation abgebildet und die Risikowerte berechnet. Die Eintretenswahrscheinlichkeit $p_{j}$ eines Szenarios ist gegeben durch die lokale Brandausbruchswahrscheinlichkeit. Diese läßt sich anhand der statistischen Analyse von tatsächlichen Startpunkten schätzen (z. B. mit logistischer Regression). Die Wahrscheinlichkeit $a_{i p}$ daß ein Brand bei Eintreten des Szenarios $j$ das Objekt $i$ erreicht, kann anhand der Brandausbreitungssimulation berechnet werden. Damit ist die Wahrscheinlichkeit $k_{i p}$ daß ein Objekt von einem Szenario betroffen wird, bekannt. Der potentielle Schaden $d_{i p}$ welcher im entsprechenden Szenario am Objekt entsteht, läßt sich mit einer geeigneten Funktion $\Phi$ aus den modellierten Brandintensitäten $I_{i j}$ berechnen.

\begin{tabular}{|l|c|c|c|c|c|c|}
\hline \multicolumn{1}{|c|}{ Szenarien } & $S_{1}$ & $\ldots$ & $S_{j}$ & $\ldots$ & $S_{m}$ & Objektbezoge- \\
Objekte: & $p_{1}$ & $\ldots$ & $p_{j}$ & $\ldots$ & $p_{m}$ & ne Risikowerte \\
\hline$O_{1}$ & & & & & & \\
\hline$\ldots$ & & & & & & \\
\hline$O_{i}$ & & & $\begin{array}{l}k_{i j}=p_{j} a_{i j} \\
d_{i j}=\Phi\left(I_{i j}\right)\end{array}$ & & & $\begin{array}{l}k_{i}=1-\prod_{j}\left(1-k_{i j}\right. \\
d_{i}=\sum_{j} k_{i j} d_{i j}\end{array}$ \\
\hline$O_{n}$ & & & & \\
\hline $\begin{array}{l}\text { Szenarienbezo- } \\
\text { gene Risikowerte }\end{array}$ & & & $\begin{array}{l}k_{j}=\sum_{i} k_{i j} \\
d_{j}=\sum_{i} k_{i j} d_{i j}\end{array}$ & & & $K=\sum_{i} k_{i}$ \\
\hline
\end{tabular}

Tabelle 2 Risikomatrix (für eine Situation).

Aus der Matrix können die folgenden Resultate abgeleitet werden (jeweils bezogen auf einen Referenzzeitraum): - Die Wahrscheinlichkeit $k_{\dot{p}}$ daß das Objekt von einem Waldbrand betroffen wird, sowie der zu erwartende Schaden $d_{i}$ für das Objekt.
- Die zu erwartende Anzahl Objekte $k_{\dot{p}}$ welche von einem Szenario betroffen werden, und der Schaden $d_{\dot{p}}$ der dabei entstehen dürfte.

- Die zu erwartende Anzahl Objekte $K$, welche in der betrachteten Situation von Waldbränden betroffen werden, und der dabei verursachte Gesamtschaden $D$.

Dieses Vorgehen kann für mehrere Situationen wiederholt werden. Dadurch können die Situationen bezüglich des Waldbrandrisikos miteinander verglichen und unter Berücksichtigung ihrer Eintretenswahrscheinlichkeit zu einer Gesamtsicht kombiniert werden.

\subsection{Umsetzung im GIS}

Bei der praktischen Umsetzung der Risikoanalyse im GIS stellen sich eine Reihe von Problemen. Das gewichtigste ergibt sich aus der kontinuierlichen Verteilung der Gefahrenquelle und der daraus resultierenden unendlichen Menge von Szenarien. Die mit der Abbildung des Untersuchungsgebiets in einem Rastermodell einhergehende Diskretisierung des Raumes scheint dieses Problem aufden ersten Blick zu lösen. Die Menge der Szenarien wird dadurch aber lediglich von Unendlich auf eine zwar endliche, aber (bei typischen Gebietsgrößen und Rasterweiten) sehr große Zahl reduziert (im folgenden Beispiel $>10^{5}$ ). Zurzeit ist es noch nicht praktikabel für diese Menge von Szenarien die benötigten Brandverhaltensmodellierungen durchzuführen. Viel günstiger wäre es, wenn die für das Füllen der Risikomatrix nötigen Berechnungen pro Objekt statt pro Szenario durchgeführt werden könnten, da die Anzahl Objekte immer wesentlich geringer sein dürfte als jene der Szenarien (im Beispiel $10^{2}$ ). Glücklicherweise ist dies tatsächlich möglich. Es läßt sich zeigen, daß im Fall der lokalen Berechnung der Ausbreitungsparameter ein Rückwärtsverfolgen der Bewegung der Feuerfront möglich ist (SCHÖNING, 1996 b). Somit kann die Ausbreitungszeit von jedem möglichen Startpunkt zu einem bestimmten Objekt und für jeden Startpunkt die beim Objekt auftretende Feuerintensität berechnet werden.

Weitere Probleme stellen sich bei der Auswahl der zu berücksichtigenden Objekte, der Berechnung des Schadens an diesen Objekten in Abhängigkeit der Brandintensität sowie der Berechnung der Ausbreitungswahrscheinlichkeit aus den Resultaten der Ausbreitungssimulation. Diese Probleme sind erst ansatzweise gelöst und erfordern weitere Arbeiten. Dennoch wurde eine erste Umsetzung der Risikoanalyse in einem Testgebiet im Malcantone vorgenommen (Abb. 5 und Abb.6). Wegen vereinfachender Annahmen, die aufgrund der noch vorhandenen Lücken in der Analysestruktur getroffen werden mußten, sollten die gezeigten Resultate allerdings sehr zurückhaltend interpretiert werden.

Abb. 5 zeigt den zu erwartenden Schaden für alle möglichen Startpunkte (die Werte $d_{j}$ aus der Risikomatrix). Die verwendete Situation entspricht einer Südwindlage. Für die gleiche Situation ist in Abb. 6 der erwartete Schaden $d_{i}$ an den einzelnen Objekten dargestellt. 


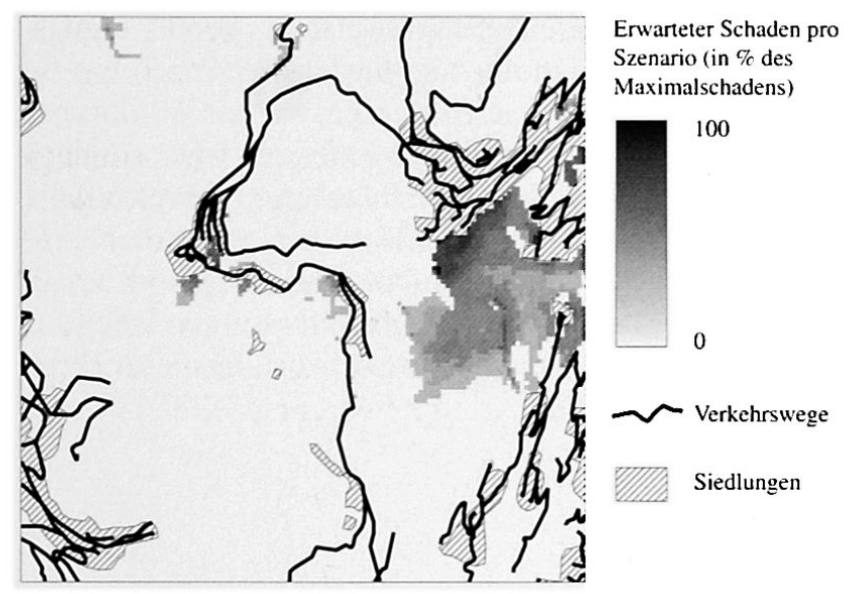

Abb. 5 Enwarteter Schaden, aufgeteilt auf die potentiellen Startpunkte.

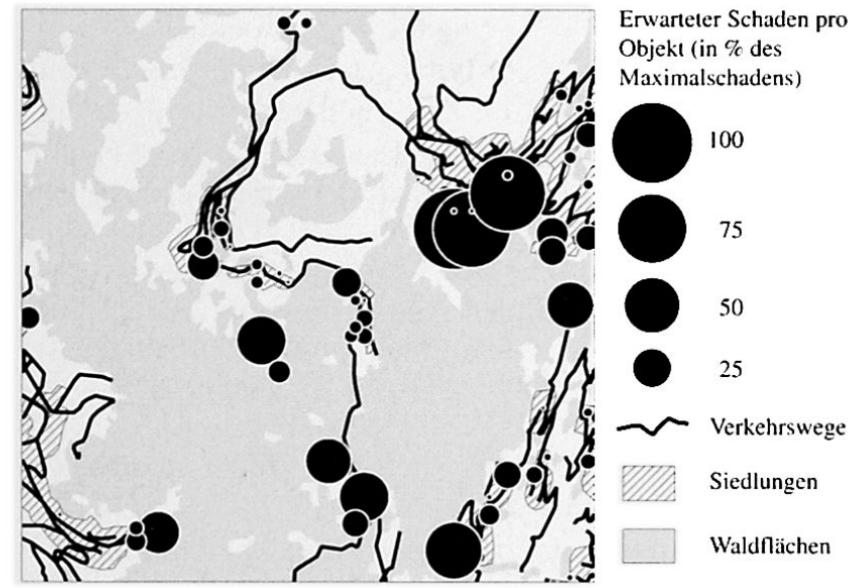

Abb. 6 Erwarteter Schaden an den gefährdeten Objekten.

\subsection{Einbezug von Bekämpfungsmaßnahmen}

Die bis anhin vorgestellte Methode der Risikoanalyse berücksichtigt den Einfluß von Managementmaßnahmen, wie das Eingreifen der Feuerwehr, noch nicht. So ist z. B. die Wahrscheinlichkeit, daß ein Feuer ein bestimmtes Objekt erreicht, nicht allein von der Ausbreitungszeit abhängig, sondern ebenso von der Interventionszeit, also der Zeit, welche verstreicht, bis die Brandbekämpfung einsetzt. BACHMANN (1996) hat ein mögliches Verfahren aufgezeigt, wie durch den Vergleich von Ausbreitungszeit und Interventionszeit Bekämpfungsmaßnahmen in die Risikoanalyse integriert werden können.

Die Ausbreitungszeit wird nach der oben erwähnten Methode der Rückwärtsverfolgung der Feuerfront für jede Zelle berechnet. Die Interventionszeit setzt sich zusammen aus der Entdeckungszeit, der Alarmierungszeit und der Anfahrtszeit (Abb. 7).

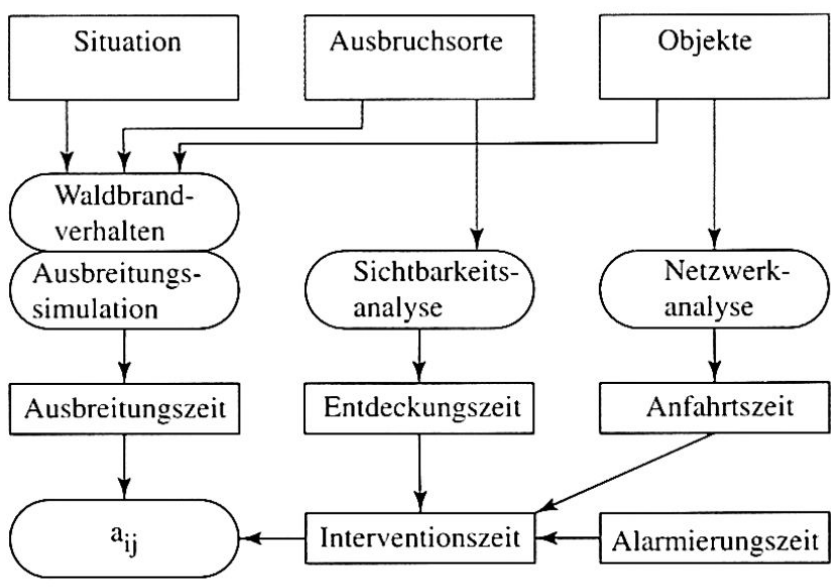

Abb. 7 Einbezug der Interventionszeit in der Risikoberechnung (Ausbreitungswahrscheinlichkeiten $a_{i j}$ ).

Die Entdeckungszeit stellt die Zeit dar, welche verstreicht, bis ein Brandausbruch erkannt wird. Diese ist abhängig von der Einsehbarkeit der potentiellen Startpunkte. Mit Hilfe der GIS-Methoden für die Sichtbarkeitsanalyse können von jedem beliebigen Beobachtungsstandpunkt aus die Gebiete bezeichnet werden, welche nicht eingesehen werden können. Die Wahl der Beobachtungsstandpunkte muß dabei speziell untersucht werden. In Frage kommen Straßen, Siedlungen, spezielle Beobachtungspunkte usw. Je nach Art des Beobachtungsstandpunktes sind unterschiedliche "Beobachtungsfrequenzen» zu berücksichtigen. Das Verkehrsaufkommen auf einer Straße kann z. B. stark abhängig sein vor der Tageszeit, vom Wochentag oder gar von der Jahreszeit. Entsprechend ist die Entdeckungszeit der Gebiete diesen Beobachtungsfrequenzen anzupassen.

Die Alarmierungszeit entspricht der Zeit, welche verstreicht, bis die Feuerwehr von ihrem Standort ausrückt. Darin inbegriffen sind das Aufsuchen einer Kommunikationsmöglichkeit zur Benachrichtigung der Feuerwehr, eventuelle Abklärungen und Einsatzplanung, Bereitstellung von Material und Bekämpfungseinheiten usw. GroBe Unterschiede dürften sich hier vor allem bei unterschiedlichen Organisationformen der Feuerwehr (Berufs- vs. freiwillige Feuerwehr) und in der Erschließung des betroffenen Gebietes (z. B. Distanz zur nächsten Telefonzelle) herausstellen.

Die Anfahrtszeit wird für jedes einzelne Objekt berechnet und entspricht der Zeit, welche die Feuerwehr braucht, um von ihrem Standort aus ein Objekt zu erreichen. Grundlage dazu ist eine umfassende Netzwerkanalyse, welche neben der einfachen Distanzberechnung weitere Faktoren wie Befahrbarkeit für verschiedene Fahrzeuge, Maximalgeschwindigkeiten oder Hindernisse berücksichtigen kann.

Die Interventionszeit stellt die Summe der Entdekkungs-, Alarmierungs- und Anfahrtszeit dar und wird für jede Zelle bestimmt. Durch den Vergleich mit der Ausbreitungszeit kann dann die Ausbreitungswahrscheinlichkeit $a_{i j}$ berechnet werden. 


\section{Schlußfolgerungen}

Geographische Informationssysteme können einen wichtigen Beitrag zu einem umfassenden und interdisziplinären Umgang mit der Waldbrandproblematik leisten. Sie ermöglichen die Integration von Daten und Modellen aus den verschiedenen beteiligten Fachrichtungen sowie die Anwendung explorativer Analyseverfahren zum Zweck des besseren Einblicks in die Problemsituation und in mögliche Lösungswege. Dieses Potential wird in der heutigen Praxis des Feuermanagements allerdings oft noch nicht voll ausgeschöpft. Die Arbeiten am Geographischen Institut der Universität Zürich versuchen deshalb, diesbezügliche Möglichkeiten aufzuzeigen und neue Ansätze zu entwickeln. In diesem Sinne ist auch die vorgestellte Methode für die Analyse des Waldbrandrisikos zu verstehen. Sie soll einen Weg aufzeigen, wie die von Waldbränden ausgehenden Gefahren systematischer und umfassender behandelt werden können. Vor einer praktischen Anwendung dieser Methode müssen indes noch eine Reihe von Fragen beantwortet werden, darunter die folgenden:

- Wie können die teilweise beträchtlichen Unsicherheiten in den Daten und Modellen bei der Berechnung und Interpretation des Waldbrandrisikos berücksichtigt werden? Für das Rothermel-Modell (ROTHERMEL 1972) wurden Versuche der Unsicherheits- und Sensitivitätsanalyse mit Monte-Carlo-Simulation durchgeführt (sCHÖNING 1996a). Entsprechende Methoden müssen insbesondere für die Ausbreitungssimulation entwickelt werden.

- Gefährdete Objekte können sowohl natürlicher wie auch menschgemachter Art sein und die verschiedensten räumlichen Ausprägungen aufweisen. Wie sollen diese Objekte repräsentativ ausgewählt und beschrieben werden?

- Wie lassen sich Schäden an den gefährdeten Objekten exakt quantifizieren? Diese Schäden können sowohl bezüglich ihrer Erscheinungsform wie auch ihrer zeitlichen Dimension sehr verschiedenartig sein. Beides macht eine einheitliche, quantitative Beurteilung, geschweige denn eine Vorhersage, sehr schwierig.

- Wie kann die Wahrscheinlichkeit der Brandausbreitung zwischen zwei Lokalitäten zuverlässiger berechnet werden? Gefragt ist vor allem die Kalibrierbarkeit anhand realer Brände bzw. der Erfahrung von Fachleuten.

Für eine wirksame Verwendung der geschilderten Risikoanalyse im Feuermanagement sollte diese auch in der Lage sein, den Einfluß von bestehenden und geplanten Managementmaßnahmen auf das Waldbrandrisiko zu simulieren. Der in 4.4 skizzierte Ansatz zur Berücksichtigung der Interventionszeit stellt einen ersten Schritt in diese Richtung dar. In Verbindung mit Verfahren der Risikobewertung könnten so kostenwirksame Maßnahmen gefunden werden, um das Waldbrandrisiko auf ein akzeptables Maß zu senken. Eine Methode für die räumliche Analyse des Waldbrandrisikos kann außer im Feuermanagement aber auch in anderen Bereichen von Nutzen sein. Zu nennen wären beispielsweise das Versicherungswesen, die Raumplanung oder die Umweltforschung. So könnte nicht nur untersucht werden, wie das Waldbrandrisiko auf gezielte Maßnahmen reagieren wird, sondern auch, wie sich Veränderungen in unserer Umwelt auf die Gefährdung durch Waldbrände auswirken werden. Angesichts der drohenden Klimaveränderung und des in vielen waldbrandbelasteten Gebieten rasanten Wechsels der Landnutzung kommt der Beantwortung dieser Frage große Bedeutung zu.

\section{Verdankungen}

Die AutorInnen möchten sich bei Marianne Rüegsegger, Stefan Harvey und dem Projekt GIS Nationalpark für die zur Verfügung gestellten Daten und bei Prof. Brassel für die Durchsicht des Manuskripts bedanken. Dieses Projekt wurde unterstützt vom Forschungsprogramm MINERVE 2 der Europäischen Union und dem Projekt GIS Nationalpark.

\section{Literatur}

ANDERSON, H. E. (1983): Predicting wind-driven wildland fire size and shape. Ogden, UT: USDA Forest Service, Intermountain Forest and Range Experiment Station.

BACHMANN, A. (1996): GIS-Analyse für ein Waldbrand-Management-System. In: Einsatz von Geographischen Informationssystemen und Fernerkundung in der Umweltanalyse. Tagungsband zum Symposium der 176. Jahresversammlung der SANW. Geoprocessing Reihe, Vol. 31.

EIDG. FORSTDIREKTION (1992): Waldbrandstatistik 1967-1991. Buwal, Abt. Wald und Holz.

HARVEY, S. (1996): Brandgutdaten in der Waldbrandmodellierung. Diplomarbeit, Geographisches Institut der Universität Zürich.

JECKLIN, R., SCHÖB, T. (1993): Waldbrandbekämpfung im Gebirge. Chur: Verlag Bündner Wald.

MERZ, H. A., SCHNEIDER, TH., BOHNENBLUST, H. (1995): Bewertung von technischen Risiken. Polyprojekt Risiko und Sicherheit, Dokument Nr. 3, ETH Zürich.

ROTHERMEL, R. C. (1972): A mathematical model for predicting fire spread in wild land fuels. Odgen, UT: USDA Forest Service, Intermountain Forest and Range Experiment Station.

RÜEGSEGGER, M. (1996): Entscheidungsunterstützung im Waldbrandmanagement mit GIS; Strategien und ausgewählte Beispiele. Diplomarbeit, Geographisches Institut der Universität Zürich.

SCHÖNING, R. (1996a): Modellierung des potentiellen Waldbrandverhaltens mit einem Geographischen Informationssystem. Diplomarbeit, Geographisches Institut der Universität Zürich

SCHÖING, R. (1996 b): Rückwärtsverfolgung der Brandausbreitung. Interner Bericht, Geographisches Institut der Universität Zürich.

SIMARD, A. J. (1976): Wildland fire management: the economics of policy alternatives: Department of Fisheries and Environment, Canadian Forestry Service. 